\title{
Predicting Optimal Combination LT4 + LT3 Therapy for Hypothyroidism Based on Residual Thyroid Function
}

\author{
Joseph DiStefano III $^{1 *}$ and Jacqueline Jonklaas ${ }^{2}$ \\ ${ }^{1}$ Departments of Computer Science and Medicine, University of California, Los Angeles, Los Angeles, CA, United States, \\ ${ }^{2}$ Division of Endocrinology, Georgetown University, Washington, DC, United States
}

OPEN ACCESS

Edited by:

Michele Marinò

University of Pisa, Italy

Reviewed by:

Marco Centanni,

Sapienza University of Rome, Italy

Gabriela Brenta,

Dr. César Milstein Care Unit, Argentina

*Correspondence:

Joseph DiStefano III

joed@cs.ucla.edu

Specialty section:

This article was submitted to

Thyroid Endocrinology,

a section of the journal

Frontiers in Endocrinology

Received: 09 August 2019 Accepted: 14 October 2019 Published: 15 November 2019

Citation:

DiStefano J III and Jonklaas J (2019) Predicting Optimal Combination LT4 + LT3 Therapy for Hypothyroidism Based on Residual Thyroid Function.

Front. Endocrinol. 10:746. doi: 10.3389/fendo.2019.00746
Objective: To gain insight into the mixed results of reported combination therapy studies conducted with levothyroxine (LT4) and liothyronine (LT3) between 1999 and 2016.

Methods: We defined trial success as improved clinical outcome measures and/or patient preference for added LT3. We hypothesized that success depends strongly on residual thyroid function (RTF) as well as the LT3 added to sufficient LT4 dosing to normalize serum T4 and TSH, all rendering T3 levels to at least middle-normal range. The THYROSIM app was used to simulate "what-if" experiments in patients and study designs corresponding to the study trials. The app graphically provided serum total (T4) and free (FT4) thyroxine, total (T3) and free (FT3) triiodothyronine, and TSH responses over time, to different simulated LT4 and combination LT4 + LT3 dosage inputs in patients with primary hypothyroidism. We compared simulation results with available study response data, computed RTF values that matched the data, classified and compared them with trial success measures, and also generated nomograms for optimizing dosages based on RTF estimates.

Results: Simulation results generated three categories of patients with different RTFs and T3 and T4 levels at trial endpoints. Four trial groups had $>20 \%$, four $<10 \%$, and five 10-20\% RTF. Four trials were predicted to achieve high, seven medium, and two low T3 levels. From these attributes, we were able to correctly predict 12 of 13 trials deemed successful or not. We generated an algorithm for optimizing dosage combinations suitable for different RTF categories, with the goal of achieving mid-range normal T4, T3 and TSH levels. RTF is estimated from TSH, T4 or T3 measurements prior to any hormone therapy treatment, using three new nonlinear nomograms for computing RTFs from these measurements. Recommended once-daily starting doses are: $100 \mu \mathrm{g}$ LT4 + 10-12.5 $\mu \mathrm{g}$ LT3; $100 \mu \mathrm{g} \mathrm{LT4} \mathrm{+} \mathrm{7.5-10} \mathrm{\mu g} \mathrm{LT3;} \mathrm{and} \mathrm{87.5} \mathrm{\mu g} \mathrm{LT4} \mathrm{+} 7.5 \mu \mathrm{g}$ LT3; for $<10 \%, 10-20 \%$, and $>20 \%$ RTF, respectively.

Conclusion: Unmeasured and variable RTF is a complicating factor in assessing effectiveness of combination LT4 + T3 therapy. We have estimated and partially validated RTFs for most existing trial data, using THYROSIM, and provided an algorithm for estimating RTF from accessible data, and optimizing patient dosing of LT4 + LT3 combinations for future combination therapy trials.

Keywords: simulation, combination therapy, levothyroxine, liothyronine, residual thyroid function, hypothyroidism etiology 


\section{INTRODUCTION}

Combination therapy for hypothyroidism using both levothyroxine (LT4) and liothyronine (LT3) continues to be a topic of much interest to physicians and patients alike (1-4). This interest has been spurred, in part, by the welldocumented finding that the ratio of total thyroxine (T4) to total triiodothyronine (T3) increases during LT4 therapy, compared with endogenous euthyroidism (5), and also that T3 levels may be lower than in the native state (6). Furthermore, animal studies suggest T3 deficiency at the tissue level with LT4 therapy alone $(7,8)$. This interest persists despite the generally mixed results of combination therapy trials, with most results not demonstrating a benefit of such therapy in terms of improvement in quality of life, mood, or neurocognitive function, but some patients expressing preference for therapy containing LT3 (9-22). When examining outcomes of either quality of life, mood, or neurocognitive function, trials fall into 3 broad categories: those showing substantial clinical benefit of combination therapy $(11,16)$, those showing partial benefit based on some outcome measures, but not others $(10,13,18,21)$, and those showing no benefit $(9,12,14,15,17,19,20,22)$. Similarly, the seven trials that examined patient preference for combination therapy can be divided into two groups: those in which patients preferred the LT3-containing therapy $(9-11,13,16)$, and those in which there was no preference $(18,22)$.

Numerous suggestions have been offered for why these combination therapy trials did not provide evidence of clinical benefits or greater patient preference. In addition to nonphysiologic thyroid hormone ratios, potential shortcomings include use of once daily LT3 therapy rather than two or three times a day therapy, or short duration trials or underpowered trials (23). Examining these trials aggregated into meta-analyses (24-26) also has not revealed benefits of combination therapy, perhaps due in part to the heterogeneity of the trial populations and methods, which include different doses of LT4 and LT3 employed, etiology of hypothyroidism, unknown degree of residual thyroid function (RTF), treatment duration, different thyrotropin (TSH), free or total T4 and T3 levels achieved in the two groups, and the outcome measures employed (23). The current work is focused primarily on degree of residual thyroid function, which we postulate may be responsible for generating quite variable responses to and perceived effects of added exogenous LT3.

The THYROSIM app (27) is a freely accessible, well-validated and mechanistically-based simulator of human thyroid hormone and TSH regulation dynamics, developed and implemented as a facile web-based and personal device application. THYROSIM has a simple and intuitive user interface for teaching and conducting simulated "what-if" experiments, graphically providing temporal dynamic responses-namely levels of serum total T4, T3, free T4 (FT4), and free T3 (FT3), as well as TSH responses over time, to various simulated system and input perturbations in $70 \mathrm{~kg}$ humans $(28,29)$. It has also been modified to predict LT4 and LT3 replacement in pediatric patients (30), used to explore TSH dynamics in primary and secondary hypothyroidism (31), and applied to LT4 bioequivalence studies
$(29,32)$. Furthermore, the utility of the app in clinical research also has been demonstrated more recently by predicting the potentially pathophysiological effects of over-the-counter thyroid supplements (33).

In order to gain insight into the mixed results of the 14 combination therapy trials, we developed the following two hypotheses to test predictively using the THYROSIM app and retrospectively using data from the trials. For both hypotheses, combination therapy is understood to mean addition of LT3 to LT4 dosing; and "success" of combination therapy was defined as benefit in terms of improved clinical outcome measures (quality of life, mood, or neurocognitive function) or patient preference for the added LT3.

\section{Working Hypothesis 1}

Success with combination therapy will be greatest when the daily LT4 dose fraction is sufficient to normalize serum TSH and T4 and the daily LT3 dose added renders serum T3 levels within the middle to upper normal range.

\section{Working Hypothesis 2}

Success with combination therapy depends strongly on a patient's RTF as well as the LT3 added to sufficient LT4 dosing. Little or no success is predicted when RTF is $20 \%$ or more unless the daily LT3 dose added generates serum T3 levels in the mid-normal to high normal T3 range. Preference for combination therapy is not likely unless the added T3 generates high-normal range to supra-physiologic T3 levels.

\section{METHODS}

\section{Dosage Response Simulations}

The THYROSIM app (27) has been applied in the current work by exploring THYROSIM responses to exogenous LT4 and combination LT4 + LT3 hormone dosage inputs in simulated patients with primary hypothyroidism, and patients with different degrees of RTF, rendered hypothyroid by autoimmune thyroid disease, radioactive iodine therapy, external beam radiotherapy, or thyroid surgery. In support of predicted results, simulation conditions - namely dosages and predicted RTFwere adjusted to and compared with data from several studies of patients receiving synthetic combination LT4 + LT3 therapy in comparison with LT4 therapy alone (9-22). An example of a simulation matching data from Siegmund et al. (20) is shown in Figure 1.

\section{RTF Measures}

To obtain RTF estimates for our data with the THYROSIM app, we simulated patient dosing input regimens and output responses with serum TSH, T4, and T3 presumed to be measured before any therapy was begun. RTF is estimated by manually adjusting the T4 and T3 secretion rates on the graphic interface of the THYROSIM app, by trial-and-error. The goal is to find the best RTF (\% secretion rates) that generates starting values (initial hormone concentrations) that approximate both the initial T4, T3, and TSH concentrations measured prior to dosing therapy (combination therapy or T4 monotherapy), and the approximate final concentrations measured at the end of the study period. 


\section{DIRECTIONS EXAMPLE DISCLAIMER Start by clicking "Show $\mathrm{T}_{3}$ input" or "Show $\mathrm{T}_{4}$ input"}
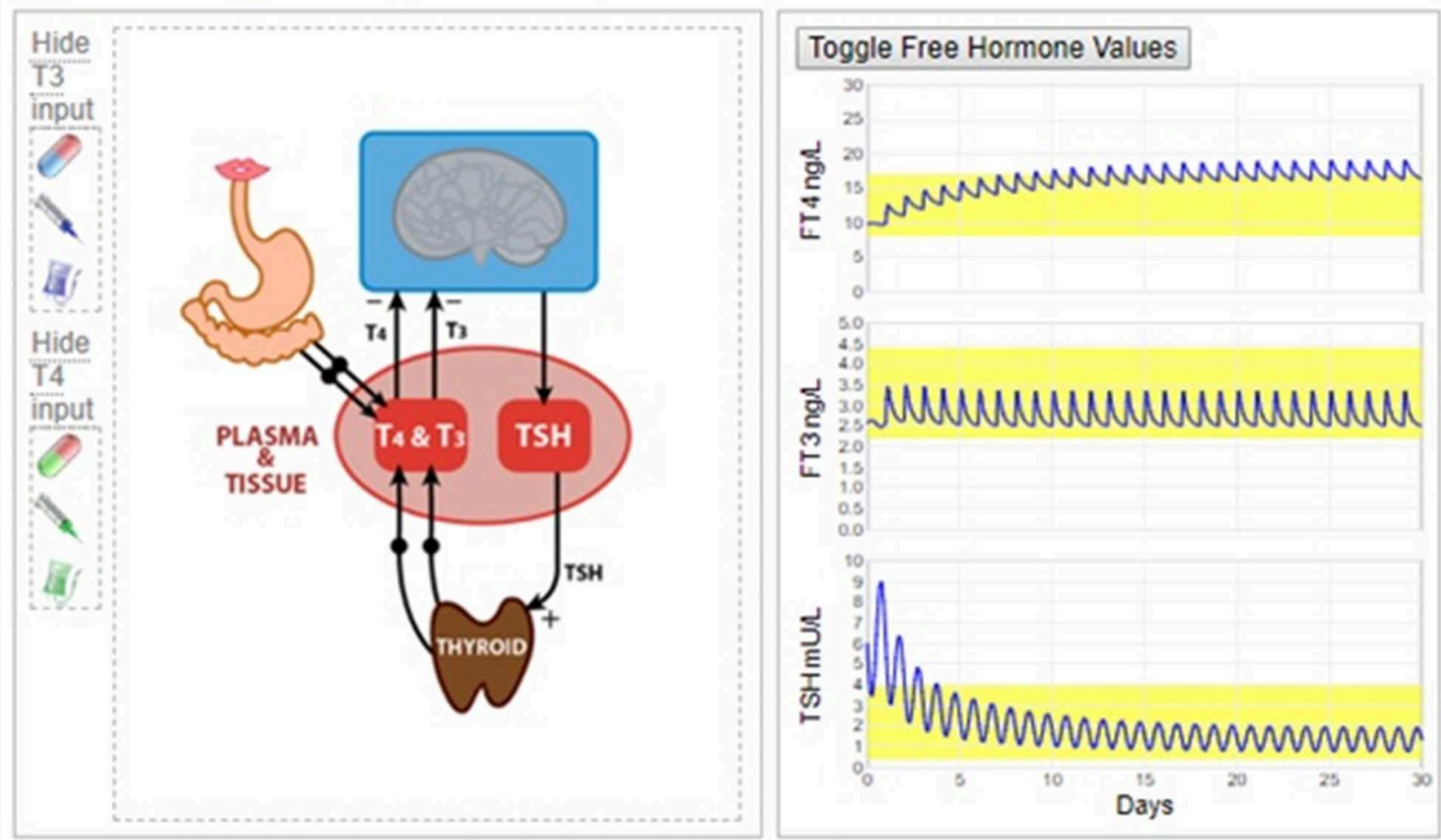

Adjust secretion/absorption rates:

\& Recalculate Initial Conditions

$\begin{array}{llll}T_{4} \text { Secretion (0-200\%): } & \begin{array}{l}T_{4} \text { Absorption (0-100\%): } \\ 25 \%\end{array} \quad \begin{array}{llll}T_{3} \text { Secretion } \\ 25\end{array} \% \text { (0-200\%): } & \begin{array}{l}T_{3} \text { Absorption }(0-100 \%): \\ 25\end{array} \%\end{array}$

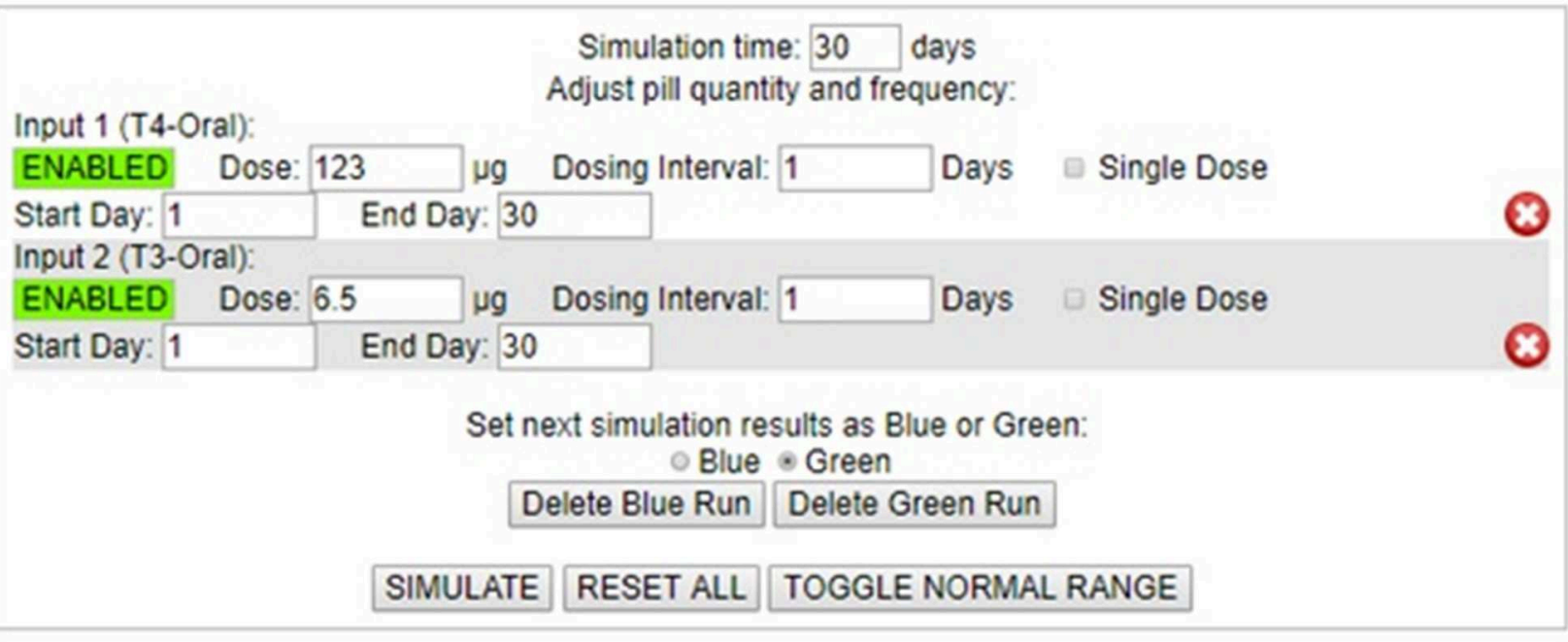

FIGURE 1 | Simulation of $123 \mu \mathrm{g} \mathrm{LT4} \mathrm{+} 6.5 \mu \mathrm{g}$ LT3 dosing experiment in Siegmund 2004 trial (20). Final hormone values achieved with 25\% RTF. 
For hypothyroid patients with different etiologies of their hypothyroidism, it is important that these thyroid variables are assessed after they reach a steady state, after they plateau, and after the degree of RTF also stabilizes. For example, following complete thyroidectomy, the thyroid hormone and TSH levels 6 weeks later should indicate $0 \%$ RTF. For someone with Hashimoto's hypothyroidism, in order to predict their likely non-zero RTF, at least 6 weeks are needed for the thyroid hormone and TSH levels to stabilize following likely incomplete thyroid destruction.

Only one study was available from among the 14 combination therapy trials that provided any measured patient hormone values prior to initiating therapy for hypothyroidism, and this was only for TSH (16). THYROSIM simulations were conducted with different RTF values, by varying the thyroidal T4 and T3 secretion rates from 0 (athyreotic) to $50 \%$ and recording the starting values for total serum TSH, T4 and T3. We assumed that $\mathrm{T} 3$ and $\mathrm{T} 4$ secretion rates (adjustable on the THYROSIM interface) are suppressed or reduced together by relatively the same amounts.

\section{RESULTS}

\section{Method Validation}

To help validate our computational modeling approach, we simulated the combination therapy dosing and dose-response conditions reported in the study of 10 patients from the Saravanan trial, which provided $24 \mathrm{~h}$ hormone profiles of TSH, FT3 and FT4 in 20 hypothyroid patients taking either LT4 monotherapy or combined LT3/LT4 therapy (34). Simulation response results (solid blue lines) are shown graphically in Figure 2, superimposed over the published data corresponding to these results. They match the data quite well. In particular, the $\sim 40 \%$ rise in mean FT3 values, peaking at $\sim 4 \mathrm{~h}$, is well represented by the simulation and is shown to remain within the normal FT3 range (yellow band), thus tracking the previously reported data well. In comparison, the Saravanan sub-study (34) reported 3 of 10 patients in the LT3/LT4 group, but none in the LT4 alone group, had FT3 levels above their laboratory reference range at some time over the 24 -h period, but lasting only for $<2 \mathrm{~h}$.

\section{Addressing the Hypotheses}

A summary of conditions, patient populations and hypothyroidism etiologies from 13 combination therapy trials (excluding Valizadeh) is given in Table 1. This table shows (where available) the LT4 doses prior to randomization, and the LT4 and LT4/LT3 doses in the monotherapy and combination therapy arms. Full information about the etiology of the hypothyroidism was not provided in all trials.

\section{Predicted TSH, T4, and T3 Levels vs. RTF Values at Diagnosis}

The three graphs shown in Figure 3 illustrate the predicted $\mathrm{TSH}, \mathrm{T} 4$, and T3 levels prior to initiating any therapy for hypothyroidism in individuals with RTF varying between $0 \%$ (athyreotic) up to 50\% RTF. The relationships are nonlinear,

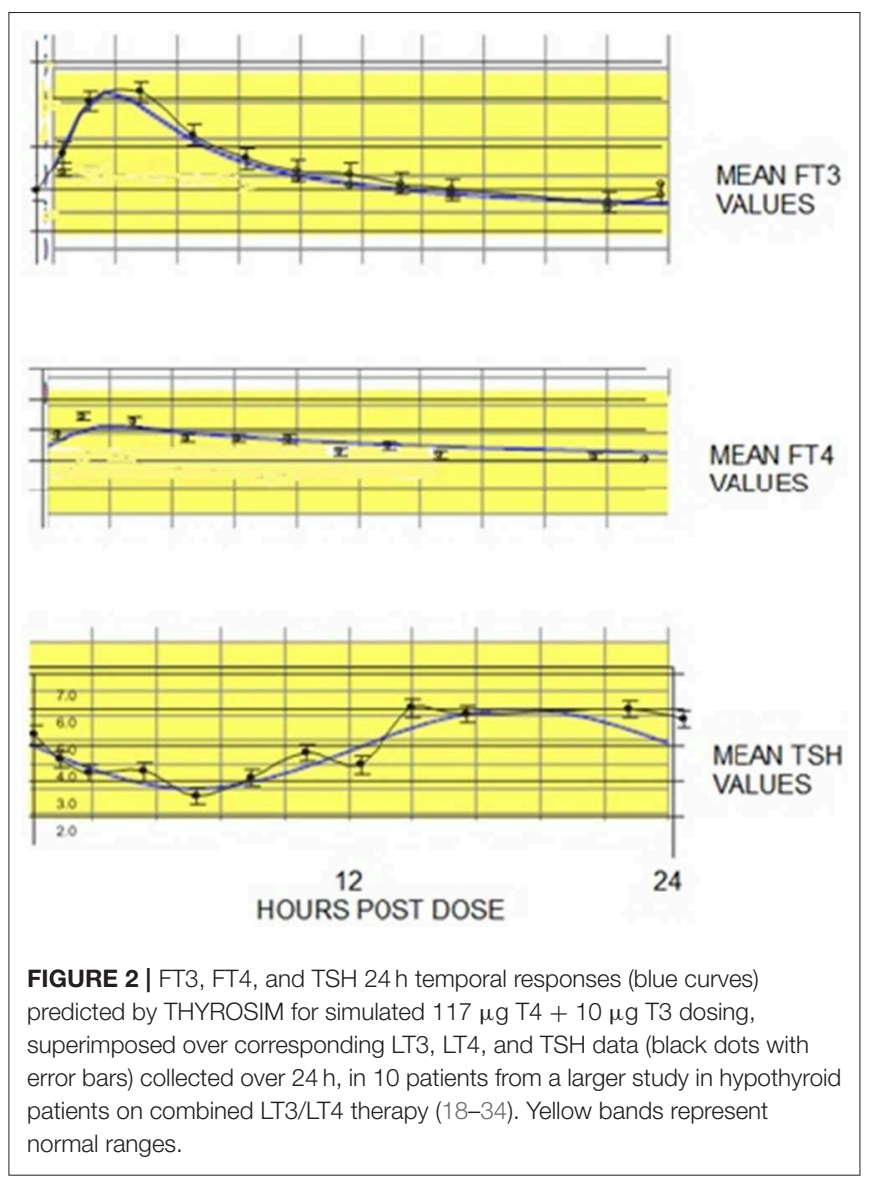

particularly in the most likely RTF range, up to 30\%; and TSHfollowed by $\mathrm{T} 4$-followed by $\mathrm{T} 3$ levels, are the most sensitive to increasing RTF.

\section{RTF Estimates and Predicted Success of LT3/LT3 Therapy \\ Patient RTF Values}

The best results predicted by THYROSIM and supported by the trial data suggest that, because the trials included patients with different etiologies of hypothyroidism, the participants had varying degrees of RTF. Table 2 shows the various trials separated into categories of simulated high, medium and low RTF values, respectively. The data in published results were incomplete, so the categories may not be completely accurate.

For the first category of high RTF ( $>20 \%$ ), no benefit of combination therapy was predicted with respect to quality of life, mood or neurocognitive benefit or LT4/LT3 preference in the 4 trials with high RTFs $(9,10,13,20)$. We predicted that with $>20 \%$ RTF, small amounts of added LT3 would have less of an impact, as assessed by various outcome measures or patient preference. We speculated that combination therapy would only be clinically successful in the setting of high RTF if the LT4 dose maintained T4 and TSH in their normal ranges and the added LT3 dose generated higher than normal T3 levels. For higher LT3 doses, 
TABLE 1 | Summary of 13 trials of synthetic combination with LT4/LT3 therapy compared to LT4 alone.

\begin{tabular}{|c|c|c|c|c|c|c|c|c|c|c|}
\hline References & $\begin{array}{l}\text { Treatment } \\
\text { dosing }\end{array}$ & Dose of LT4 pre-trial & $\begin{array}{l}\text { Dose of } \\
\text { LT4 in LT4 } \\
\text { gp } \\
\text { (number } \\
\text { of } \\
\text { patients) }\end{array}$ & $\begin{array}{l}\text { Dose of LT4 in } \\
\text { LT3 gp } \\
\text { (number of } \\
\text { patients) }\end{array}$ & $\begin{array}{l}\text { Dose of LT3 in } \\
\text { LT3 gp }\end{array}$ & $\begin{array}{l}\text { Etiology } \\
\text { primary hypo- } \\
\text { thyroidism } \\
\text { (number of } \\
\text { patients) }\end{array}$ & Design & $\begin{array}{l}\text { Number of } \\
\text { patients } \\
\text { randomized } \\
\text { (completed } \\
\text { follow-up) }\end{array}$ & $\begin{array}{l}\text { Treatment } \\
\text { duration }\end{array}$ & $\begin{array}{l}\text { Baseline \& end of } \\
\text { study TSH } \\
\text { differences between } \\
\text { groups }\end{array}$ \\
\hline Appelhof et al. (9) & $\begin{array}{l}\text { T4: usual } \\
\text { dose } \\
\text { LT4/LT3: 10:1 } \\
\text { or 5:1 ratio of } \\
\text { T4 to T3 ratio, } \\
\text { respectively } \\
\text { Dosing: Twice } \\
\text { daily for both } \\
\text { LT4 \& LT3 }\end{array}$ & $\begin{array}{l}1.46 \mu \mathrm{g} / \mathrm{kg} / \text { day (placebo) } \\
1.61 \mu \mathrm{g} / \mathrm{kg} / \text { day (LT4: LT3 } \\
10: 1) \\
1.73 \mu \mathrm{g} / \mathrm{kg} / \text { day (LT4: } \\
\text { LT3 5:1) }\end{array}$ & $\begin{array}{l}100 \mu \mathrm{g}(50 \\
\mu \mathrm{g} \text { given } \\
\text { twice daily) }\end{array}$ & $\begin{array}{l}75 \mu \mathrm{g}(10: 1 \\
\text { ratio) } \\
75 \mu \mathrm{g}(5: 1 \text { ratio) } \\
\text { (approx. } 37.5 \mu \mathrm{g} \\
\text { given twice daily) }\end{array}$ & $\begin{array}{l}7.5 \mu \mathrm{g}(3.75 \mu \mathrm{g} \\
\text { twice daily) } \\
(10: 1) \\
15 \mu \mathrm{g} 7.5 \mu \mathrm{g} \\
\text { twice daily } \\
(5: 1)\end{array}$ & $\begin{array}{l}\text { Autoimmune } \\
\text { (other causes } \\
\text { excluded), 80\% } \\
\text { positive TPO } \\
\text { antibodies }\end{array}$ & $\begin{array}{l}\text { Parallel, } \\
\text { blinded }\end{array}$ & $141(130)$ & 15 weeks & $\begin{array}{l}\text { Baseline TSH values } \\
\text { 1-1.1. LT4 vs. LT4/LT3 } \\
\text { (10:1) vs. LT4/LT3 (5:1) } \\
0.64 \text { vs. } 0.35 \text { vs. } 0.07 \\
\text { (TSH lower in the } 5: 1 \\
\text { T3:T4 dose group) }\end{array}$ \\
\hline $\begin{array}{l}\text { Bunevicius et al. } \\
\text { (11) }\end{array}$ & $\begin{array}{l}\text { T4: usual } \\
\text { LT4/LT3: } \\
\text { usual T4 dose } \\
\text { minus } 50 \\
\mu \mathrm{g} / \text { day with } \\
\text { T3 } 12.5 \\
\mu \mathrm{g} / \text { day } \\
\text { Dosing: } \\
\text { Once daily }\end{array}$ & $\begin{array}{l}175 \mu \mathrm{g} \text { (all) } \\
181 \mu \mathrm{g} \text { (placebo first) } \\
169 \mu \mathrm{g} \text { (LT3 first) }\end{array}$ & $175 \mu \mathrm{g}$ & $125 \mu \mathrm{g}$ & $12.5 \mu \mathrm{g}$ & $\begin{array}{l}\text { Mixed- } \\
\text { Autoimmune } \\
\text { (16), thyroid } \\
\text { cancer (17) }\end{array}$ & $\begin{array}{l}\text { Cross-over, } \\
\text { blinded }\end{array}$ & $35(33)$ & 5 weeks & $\begin{array}{l}\text { Baseline TSH 0.3-1.5. } \\
\text { LT4 0.8 vs. LT4/LT3 } \\
0.5 \text {. } \\
\text { NS ‡ difference }\end{array}$ \\
\hline $\begin{array}{l}\text { Bunevicius et al. } \\
\text { (10) }\end{array}$ & $\begin{array}{l}\text { T4: usual } \\
\text { LT4/LT3: } \\
\text { usual T4 dose } \\
\text { minus } 50 \\
\mu \mathrm{g} / \text { day with } \\
\text { T3 } 10 \mu \mathrm{g} / \text { day } \\
\text { Dosing: } \\
\text { Once daily }\end{array}$ & $\begin{array}{l}\text { All: } \\
100 \mu g(7) \\
150 \mu g(3)\end{array}$ & $\begin{array}{l}115 \mu \mathrm{g} \\
\text { (approx.) }\end{array}$ & $65 \mu \mathrm{g}$ (approx.) & $10 \mu \mathrm{g}$ & $\begin{array}{l}\text { All Graves } \\
\text { disease, history } \\
\text { of subtotal } \\
\text { thyroidectomy }\end{array}$ & $\begin{array}{l}\text { Cross-over, } \\
\text { blinded }\end{array}$ & $13(10)$ & 5 weeks & $\begin{array}{l}\text { Baseline TSH 1.02. LT4 } \\
0.45 \text { vs. LT4/LT3 0.47. } \\
\text { NS } \text { difference }\end{array}$ \\
\hline Clyde et al. (12) & $\begin{array}{l}\text { T4: usual } \\
\text { LT4/LT3: } \\
\text { usual T4 dose } \\
\text { minus } 50 \\
\mu \mathrm{g} / \text { day with } \\
\text { T3 } 15 \mu \mathrm{g} / \mathrm{d} \\
\text { Dosing: Twice } \\
\text { daily LT3, LT4 } \\
\text { once daily }\end{array}$ & $\begin{array}{l}131 \mu \mathrm{g} \text { (placebo) } \\
136 \mu \mathrm{g} \text { (LT3) } \\
1.6 \mu \mathrm{g} / \mathrm{kg} / \text { day (placebo) } \\
1.8 \mu \mathrm{g} / \mathrm{kg} / \text { day (LT3) }\end{array}$ & $\begin{array}{l}131 \mu \mathrm{g} \\
\text { (including } \\
25 \mu \mathrm{g} \mathrm{BID,} \\
\text { balance } \\
\text { given once } \\
\text { daily) }\end{array}$ & $86 \mu \mathrm{g}$ once daily & $\begin{array}{l}15 \mu \mathrm{g}(7.5 \mu \mathrm{g} \\
\text { twice daily) }\end{array}$ & $\begin{array}{l}\text { Mixed - } \\
\text { Autoimmune } \\
\text { (31), post-RAl }{ }^{\star} \\
\text { (10), thyroid } \\
\text { surgery (1), } \\
\text { post-EBRT } \\
\text { thyroid cancer } \\
\text { (1) }\end{array}$ & $\begin{array}{l}\text { Parallel, } \\
\text { blinded }\end{array}$ & $46(44)$ & 4 months & $\begin{array}{l}\text { Baseline TSH 2.2-2.6. } \\
\text { LT4 2.1 vs. LT4/LT3 } \\
\text { 2.0. NS \& difference }\end{array}$ \\
\hline $\begin{array}{l}\text { Escobar-Morreale } \\
\text { et al. (13) }\end{array}$ & $\begin{array}{l}\text { T4: } 100 \\
\mu \mathrm{g} / \text { day } \\
\text { LT4/LT3: LT4 } \\
75 \mu \mathrm{g} / \text { day } \\
\text { and T3 } 5 \\
\mu \mathrm{g} / \mathrm{d} \\
\text { Dosing: } \\
\text { Once daily }\end{array}$ & $100 \mu \mathrm{g}$ (all) & $100 \mu \mathrm{g}$ & $\begin{array}{l}75 \mu \mathrm{g} \\
87.5 \mu \mathrm{g} \text { (add on) }\end{array}$ & $\begin{array}{l}5 \mu \mathrm{g} \\
7.5 \mu \mathrm{g} \\
\text { (add on) }\end{array}$ & $\begin{array}{l}\text { Mixed - } \\
\text { Autoimmune } \\
\text { (23), post-RAl } \\
\text { (5) }\end{array}$ & $\begin{array}{l}\text { Cross-over, } \\
\text { blinded }\end{array}$ & $28(26)$ & 8 weeks & $\begin{array}{l}\text { Baseline TSH "normal". } \\
\text { LT4 1.95 vs. LT4/LT3 } \\
\text { 2.56. LT4/LT3 > LT4 }\end{array}$ \\
\hline
\end{tabular}


TABLE 1 | Continued

\begin{tabular}{|c|c|c|c|c|c|c|c|c|c|c|}
\hline References & $\begin{array}{l}\text { Treatment } \\
\text { dosing }\end{array}$ & Dose of LT4 pre-trial & $\begin{array}{l}\text { Dose of } \\
\text { LT4 in LT4 } \\
\text { gp } \\
\text { (number } \\
\text { of } \\
\text { patients) }\end{array}$ & $\begin{array}{l}\text { Dose of LT4 in } \\
\text { LT3 gp } \\
\text { (number of } \\
\text { patients) }\end{array}$ & $\begin{array}{l}\text { Dose of LT3 in } \\
\text { LT3 gp }\end{array}$ & $\begin{array}{l}\text { Etiology } \\
\text { primary hypo- } \\
\text { thyroidism } \\
\text { (number of } \\
\text { patients) }\end{array}$ & Design & $\begin{array}{l}\text { Number of } \\
\text { patients } \\
\text { randomized } \\
\text { (completed } \\
\text { follow-up) }\end{array}$ & $\begin{array}{l}\text { Treatment } \\
\text { duration }\end{array}$ & $\begin{array}{l}\text { Baseline \& end of } \\
\text { study TSH } \\
\text { differences between } \\
\text { groups }\end{array}$ \\
\hline Fadeyev et al. (14) & 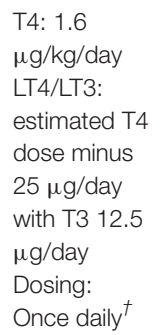 & 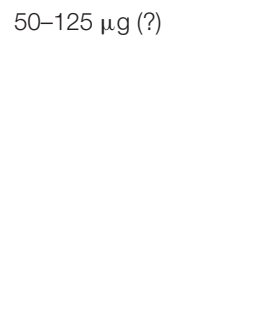 & $\begin{array}{l}100 \mu g \\
(25) \\
125 \mu g(7) \\
75 \mu g(9) \\
50 \mu g(1)\end{array}$ & $\begin{array}{l}75 \mu \mathrm{g} \text { (median) } \\
75 \mu \mathrm{g}(10) \\
100 \mu \mathrm{g}(4) \\
50 \mu \mathrm{g}(2)\end{array}$ & $12.5 \mu \mathrm{g}$ & All autoimmune & $\begin{array}{l}\text { Parallel, } \\
\text { unblinded }\end{array}$ & 58 (58?) & 6 months & $\begin{array}{l}\text { Baseline TSH "normal". } \\
\text { LT4 } 1.35 \text { vs. LT4/LT3 } \\
\text { 1.7. NS } \ddagger \text { difference }\end{array}$ \\
\hline Kaminski et al. (15) & $\begin{array}{l}\text { T4: } 125 \text { or } \\
150 \mu \mathrm{g} \\
\text { LT4/LT3 } 75 \\
\mu \mathrm{g}+15 \mu \mathrm{g} \\
\text { T3 } \\
\text { Once daily }\end{array}$ & 125 or $150 \mu \mathrm{g}$ & $\begin{array}{l}125 \text { or } 150 \\
\mu g\end{array}$ & $75 \mu \mathrm{g}$ & $15 \mu \mathrm{g}$ & $\begin{array}{l}\text { Mixed - } \\
\text { Autoimmune } \\
\text { (23), post-RAl* } \\
\text { (3), thyroid } \\
\text { cancer (6) }\end{array}$ & $\begin{array}{l}\text { Cross-over, } \\
\text { blinded }\end{array}$ & 32 & 8 weeks & $\begin{array}{l}\text { Baseline TSH 0.31. LT4 } \\
0.19 \text { vs. LT4/LT3 0.64 } \\
\text { NS ‡ difference }\end{array}$ \\
\hline Nygaard et al. (16) & $\begin{array}{l}\text { T4: usual } \\
\text { LT4/LT3: } \\
\text { usual T4 dose } \\
\text { minus } 50 \\
\mu \mathrm{g} / \text { day with } \\
\text { T3 } 20 \text { or } 50 \\
\mu \mathrm{g} / \text { day, } \\
\text { respectively } \\
\text { Dosing: } \\
\text { Once daily }\end{array}$ & $129 \mu \mathrm{g}$ (all) & $131 \mu \mathrm{g}$ & $77 \mu \mathrm{g}$ & $20 \mu \mathrm{g}$ & $\begin{array}{l}\text { Autoimmune (all } \\
\text { positive TPO } \\
\text { antibodies) }\end{array}$ & $\begin{array}{l}\text { Cross-over, } \\
\text { blinded }\end{array}$ & $68(59)$ & 12 weeks & $\begin{array}{l}\text { Median TSH at } \\
\text { diagnosis 43-82, } \\
\text { baseline TSH 1.1, LT4 } \\
0.99 \text { vs. LT4/LT3 } 0.76 . \\
\text { NS } \ddagger \text { difference }\end{array}$ \\
\hline $\begin{array}{l}\text { Rodriguez et al. } \\
\text { (17) }\end{array}$ & $\begin{array}{l}\text { T4: usual } \\
\text { LT4/LT3: } \\
\text { usual T4 dose } \\
\text { minus } 50 \\
\mu \mathrm{g} / \text { day with } \\
\text { T3 } 10 \mu \mathrm{g} / \text { day } \\
\text { Dosing: } \\
\text { Once daily }{ }^{\dagger}\end{array}$ & $\begin{array}{l}121 \mu \mathrm{g} \text { (all) } \\
118 \mu \mathrm{g} \text { (seq1, placebo) } \\
121 \mu \mathrm{g} \text { (seq2, LT3) }\end{array}$ & $118 \mu \mathrm{g}$ & $\begin{array}{l}121-50 \mu g=71 \\
\mu g\end{array}$ & $10 \mu \mathrm{g}$ & $\begin{array}{l}\text { Mixed- } \\
\text { Autoimmune } \\
(23), \text { post-RAl* } \\
\text { (4), thyroid } \\
\text { surgery (3) }\end{array}$ & $\begin{array}{l}\text { Cross-over, } \\
\text { blinded }\end{array}$ & $30(27)$ & 6 weeks & $\begin{array}{l}\text { Baseline TSH 1.7-1.8. } \\
\text { LT4 2.5-2.9 vs. } \\
\text { LT4/LT3 3.3-7.6. NS } \ddagger \\
\text { difference }\end{array}$ \\
\hline
\end{tabular}


TABLE 1 | Continued

\begin{tabular}{|c|c|c|c|c|c|c|c|c|c|c|}
\hline References & $\begin{array}{l}\text { Treatment } \\
\text { dosing }\end{array}$ & Dose of LT4 pre-trial & $\begin{array}{l}\text { Dose of } \\
\text { LT4 in LT4 } \\
\text { gp } \\
\text { (number } \\
\text { of } \\
\text { patients) }\end{array}$ & $\begin{array}{l}\text { Dose of LT4 in } \\
\text { LT3 gp } \\
\text { (number of } \\
\text { patients) }\end{array}$ & $\begin{array}{l}\text { Dose of LT3 in } \\
\text { LT3 gp }\end{array}$ & $\begin{array}{l}\text { Etiology } \\
\text { primary hypo- } \\
\text { thyroidism } \\
\text { (number of } \\
\text { patients) }\end{array}$ & Design & $\begin{array}{l}\text { Number of } \\
\text { patients } \\
\text { randomized } \\
\text { (completed } \\
\text { follow-up) }\end{array}$ & $\begin{array}{l}\text { Treatment } \\
\text { duration }\end{array}$ & $\begin{array}{l}\text { Baseline \& end of } \\
\text { study TSH } \\
\text { differences between } \\
\text { groups }\end{array}$ \\
\hline $\begin{array}{l}\text { Saravanan et al. } \\
\text { (18) }\end{array}$ & $\begin{array}{l}\text { T4: usual } \\
\text { LT4/LT3: } \\
\text { usual T4 dose } \\
\text { minus } 50 \\
\mu \mathrm{g} / \text { day with } \\
\text { T3 } 10 \mu \mathrm{g} / \text { day } \\
\text { Dosing: } \\
\text { Once daily }\end{array}$ & $\begin{array}{l}123 \mu \mathrm{g} \text { (placebo) } \\
127 \mu \mathrm{g} \text { (LT3) }\end{array}$ & $123 \mu \mathrm{g}$ & $\begin{array}{l}127-50 \mu g=77 \\
\mu g\end{array}$ & $10 \mu \mathrm{g}$ & $\begin{array}{l}\text { Primary } \\
\text { hypothyroidism } \\
\text { (72\%?, 44\% } \\
\text { TPO antibodies), } \\
\text { no thyroid } \\
\text { cancer }\end{array}$ & $\begin{array}{l}\text { Parallel, } \\
\text { blinded }\end{array}$ & 697 (573) & $\begin{array}{l}12 \text { Months } \\
\text { (outcomes } \\
\text { assessed } 3 \\
\text { and } 12 \\
\text { months) }\end{array}$ & $\begin{array}{l}\text { Baseline TSH } \\
0.84-0.85 . \text { LT4 } 0.79 \text { vs. } \\
\text { LT4/LT3 } 1.25 \text { at } 12 \\
\text { months. LT4/LT3 > LT4 } \\
\text { at } 3 \text { months }\end{array}$ \\
\hline Sawka et al. (19) & $\begin{array}{l}\text { T4: usual } \\
\text { LT4/LT3: } 50 \% \\
\text { usual T4 dose } \\
\text { with T3 total } \\
25 \mu \mathrm{g} / \text { day } \\
\text { (12.5 } \mu \mathrm{g} \text { BID) } \\
\text { Dosing: Twice } \\
\text { daily T3, once } \\
\text { daily T4 }\end{array}$ & $\begin{array}{l}120 \mu \mathrm{g} \text { (placebo) } \\
132 \mu \mathrm{g} \text { (LT3) }\end{array}$ & $118 \mu \mathrm{g}$ & $67 \mu \mathrm{g}$ & $\begin{array}{l}19 \mu \mathrm{g}(9.5 \mu \mathrm{g} \\
\text { twice daily) }\end{array}$ & $\begin{array}{l}\text { Primary } \\
\text { hypothyroidism, } \\
\text { excluded: } \\
\text { thyroid cancer, } \\
\text { history of } \\
\text { hyperthyroidism, } \\
\text { thyroidectomy }\end{array}$ & $\begin{array}{l}\text { Parallel, } \\
\text { blinded }\end{array}$ & $40(33)$ & 15 weeks & $\begin{array}{l}\text { Baseline TSH 1.75-2.2. } \\
\text { LT4 1.7 vs. LT4/LT3 } \\
\text { 1.8. NS } \ddagger \text { difference }\end{array}$ \\
\hline $\begin{array}{l}\text { Siegmund et al. } \\
\text { (20) }\end{array}$ & $\begin{array}{l}\text { T4: usual } \\
\text { LT4/LT3: } \\
\text { usual T4 dose } \\
\text { minus 5\% } \\
\text { with T3 5\% } \\
\text { (aim 14:1 ratio } \\
\text { LT4 to T3) } \\
\text { Dosing: } \\
\text { Once daily }{ }^{\dagger}\end{array}$ & $\begin{array}{l}100 \mu g(5) \\
125 \mu g(12) \\
150 \mu g(8) \\
175 \mu g(1)\end{array}$ & $129 \mu \mathrm{g}$ & $123 \mu \mathrm{g}$ & $6.5 \mu \mathrm{g}$ & $\begin{array}{l}\text { Mixed - } \\
\text { Autoimmune (2), } \\
\text { post-RAl* or }_{\text {thyroid }} \\
\text { surgery (24) }\end{array}$ & $\begin{array}{l}\text { Cross-over, } \\
\text { blinded }\end{array}$ & $26(23)$ & 12 weeks & $\begin{array}{l}\text { Baseline TSH 1.72. LT4 } \\
1.5 \text { vs LT4/LT3 0.5. } \\
\text { LT4/LT3< LT4 }\end{array}$ \\
\hline Walsh et al. (22) & $\begin{array}{l}\text { T4: usual } \\
\text { LT4/LT3: } \\
\text { usual T4 dose } \\
\text { minus } 50 \\
\mu \mathrm{g} / \text { day with } \\
\text { T3 } 10 \mu \mathrm{g} / \text { day } \\
\text { Dosing: } \\
\text { Once daily }{ }^{\dagger}\end{array}$ & $136 \mu \mathrm{g}$ & $136 \mu \mathrm{g}$ & $86 \mu \mathrm{g}$ & $10 \mu \mathrm{g}$ & $\begin{array}{l}\text { Mixed- } \\
\text { Autoimmune } \\
\text { (94), post-RAl }{ }^{*} \\
\text { (4), thyroid } \\
\text { surgery (12), no } \\
\text { thyroid cancer }\end{array}$ & $\begin{array}{l}\text { Cross-over, } \\
\text { blinded }\end{array}$ & $110(101)$ & 10 weeks & $\begin{array}{l}\text { Baseline TSH 1.3-1.5. } \\
\text { LT4 1.5 vs. LT4/LT3 } \\
\text { 3.1. LT4/LT3 > LT4 }\end{array}$ \\
\hline
\end{tabular}

${ }^{\dagger}$ Dosing not reported, assume once daily. 


\section{TSH vs RESIDUAL THYROID FCN}

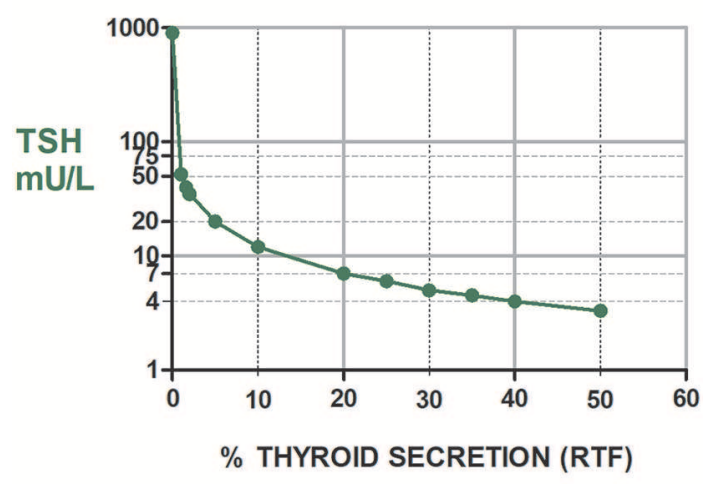

T4 vs RESIDUAL THYROID FCN

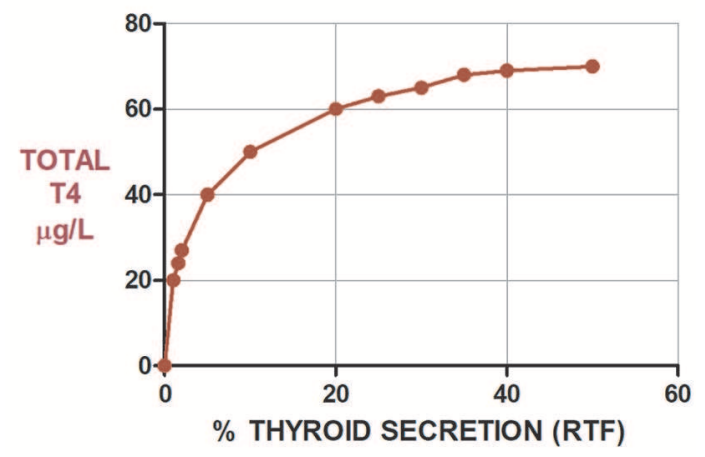

T3 vs RESIDUAL THYROID FCN

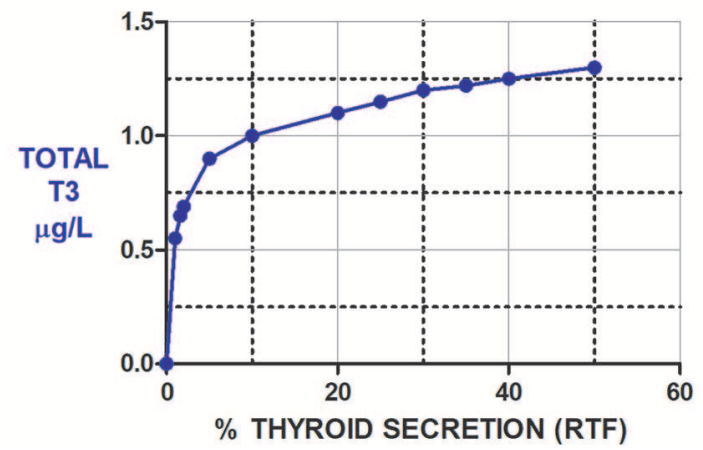

FIGURE 3 | Illustration of the predicted (and non-linear) TSH, T4, and T3 levels prior to initiating any therapy for hypothyroidism in individuals with RTFs varying between 0\% (athyreotic) up to 50\% RTF (hemi-thyroidectomized). Notably, as RTF increases from about 1-10\%, TSH levels fall by $80 \%$, while T4 levels increase by $\sim 163 \%$ and T3 levels increase by 79\%. Similarly, T4 increases by $\sim 30 \%$, T3 by $20 \%$ and TSH falls about $58 \%$ as RTF increases from 10 to $30 \%$. TSH levels shown in green, T4 levels shown in red, and T3 levels shown in blue.

the effect became noticeable and combination therapy was more likely to be preferred, albeit potentially toxic.

For the second category of medium RTF (10-20\%), some benefit with respect to quality of life or mood or neurocognitive benefit was predicted in the five relevant trials $(12,14,15,17$,
19). However, the impact of the modest amount of added LT3 on outcome measures was expected to be minimized by the endogenous RTF. If RTF was low $(<10 \%)$, combination therapy was predicted to provide substantial quality of life or mood or neurocognitive benefit and/or to be preferred by patients in the 4 relevant trials $(11,16,18,22)$.

\section{Successful Therapy Based on Improved Outcome Measures}

Table 3 shows the same 13 trials separated into three categories: (a) those showing substantial improvement in outcomes with combination therapy $(11,16)$; (b) those showing partial benefit based on some outcome measures, but not others $(13,18)$; and (c) those showing no benefit $(9,12,14,15,17,19,20,22)$. These same trials are also shown in Figures 4A-C, which categorize the trials by RTF and show the associated trial results displayed as Venn diagrams.

\section{Successful Therapy Based on Treatment Preference}

Treatment preference was assessed in 7 of the 13 trials. Table 4 lists these trials in two categories: (a) trials in which a preference for combination therapy was expressed by participating patients (9-11, 13, 16); and (b) trials in which there was no patient preference for combination therapy $(18,22)$. These trials are also indicated in Figures $\mathbf{4 A - C}$, showing how preference is related to degree of RTF, and whether either improved outcomes, preference, or both improved outcomes and preference were demonstrated in the same trial. Additionally, indicated on the figure is whether the T3 levels were predicted to be low, medium or high during the trial.

\section{Testing Hypothesis 1}

For our first hypothesis that achievement of medium-high T3 levels along with sufficient LT4 in the dose is needed for successful (improved outcomes or preference) combination therapy, our prediction was mostly correct. The Appelhof, Bunevicius, and Nygaard studies $(9-11,16)$ were predicted to have high T3 levels and were "successful" (see Figures 4A,C). The Escobar-Morreale, Kaminski, Clyde, Sawka, Rodriguez, Saravanan, and Fadeyev trials (12-15, 17-19) (Figures 4A-C) were predicted to have medium T3 levels and therefore the RTF might also impact their success. The Walsh and Siegmund trials $(20,22)$ (Figures $4 \mathbf{A}, \mathbf{C}$ ) were predicted to have low or low medium T3 levels and did not report improved outcomes or preference.

\section{Testing Hypothesis 2}

With respect to our second hypothesis of the degree of RTF (while also taking the T3 levels achieved into account) affecting the success of combination therapy, results of this prediction are shown in Figure 4. Figure 4A shows the studies with high RTF and three out of four studies are correctly predicted as not showing combination therapy to be successful. Figure 4B shows the studies with medium RTF and all five studies are correctly predicted as not showing combination therapy to be successful. For the prediction that low RTF would be associated with successful combination therapy due to the more noticeable 
TABLE 2 | Measured mean TSH, FT4, T4, T3, FT3 values at beginning and end of trials for monotherapy vs. combination therapy groups in trials grouped according to whether patients were estimated to have high RTF (>20\%), medium RTF (10-20\%), or low RTF ( $<10 \%)$.

\begin{tabular}{|c|c|c|c|c|c|c|c|c|c|c|c|}
\hline Trial & $\begin{array}{l}\text { TSH } \\
\text { before }\end{array}$ & TSH end & $\begin{array}{l}\mathrm{FT4}(\mathrm{ng} / \mathrm{dl}) \\
\text { before }\end{array}$ & FT4 end & $\begin{array}{l}\text { T3 }(\mathrm{ng} / \mathrm{dl}) \\
\text { before }\end{array}$ & T3 end & $\begin{array}{l}\mathrm{FT} 3(\mathrm{pg} / \mathrm{dl}) \\
\text { before }\end{array}$ & FT3 end & $\begin{array}{l}\text { T3 \& } T 4 \text { ranges } \\
\text { by sim }\end{array}$ & $\begin{array}{l}\text { LT3 dose } \\
(\mu \mathrm{g})\end{array}$ & $\begin{array}{l}\text { LT4 dose } \\
(\mu \mathrm{g})\end{array}$ \\
\hline \multicolumn{12}{|l|}{ High RTF (>20\%) } \\
\hline $\begin{array}{l}\text { Appelhof T4 + T3 } \\
(10: 1)\end{array}$ & 1.1 & 0.35 & 1.15 & 1.02 & 109 & 119 & - & - & Med Med & 7.5 & 75 \\
\hline Appelhof T4 + T3 (5:1) & 1.0 & 0.07 & 1.18 & 1.00 & 115 & 143 & - & - & Med-High Med & 12.5 & 75 \\
\hline $\begin{array}{l}\text { Bunevicius, 2002, T4 + } \\
\text { T3 }\end{array}$ & -1.02 & 0.47 & 1.61 & 0.96 & - & 247 & - & - & High High & 10 & 65 \\
\hline Escobar-Morreale T4 & $\mathrm{nl}$ & 1.95 & - & 1.61 & - & - & - & 332 & - & & \\
\hline $\begin{array}{l}\text { Escobar-Morreale T4 + } \\
\text { T3 }(5 \mu \mathrm{g})\end{array}$ & & 2.56 & - & 1.31 & - & - & - & 325 & Med Med & 5 & 75 \\
\hline $\begin{array}{l}\text { Escobar-Morreale T4 + } \\
\text { T3 }(7.5 \mu \mathrm{g})\end{array}$ & & 1.09 & - & 1.34 & - & - & - & 384 & Med Med & 7.5 & 87.5 \\
\hline
\end{tabular}

Medium RTF (10-20\%)

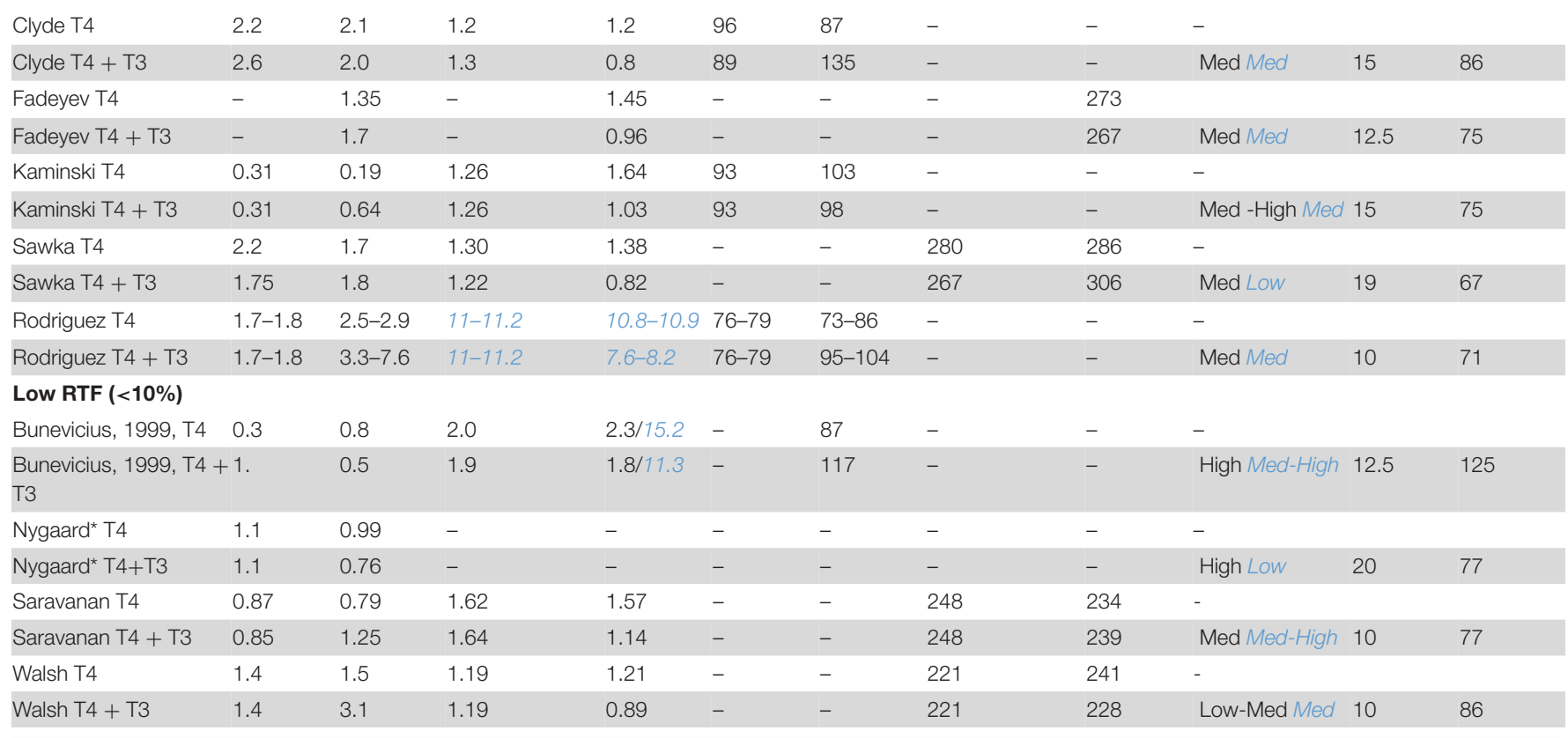

Gray shading indicates T4/T3 arm of study, "study reports only free T4 index and FT3 index and does not report either total or free T4 or T3, blue font is total T4 levels in mcg/dL.

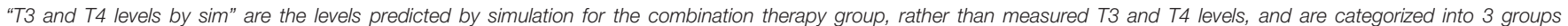
(high/medium/low). Mean/median LT3 and LT4 doses in the combination therapy group are also shown.

effect of the added LT3, we showed in Figure 4C that four out of four studies were correctly predicted in this category.

\section{Recommendations for Combination Therapy Dosing in Patients Previously Untreated With T4 or T3}

Our estimates of RTF allow us to make predictions regarding the dosing of LT3 that should be optimal when designing a combination therapy trial. Serum levels of TSH, T4 and/or T3 should be obtained at the time of diagnosis, either from patient history data or anew, prior to initiating any therapy and one or more (preferably $>1$ ) of the graphs in Figure 3 can then be used as nomograms to estimate RTF. We would then predict that the following practical daily dosing combinations would serve best for starting dosing in $70 \mathrm{~kg}$ individuals with computed RTFs in the three given ranges. (These recommended dosages should be adjusted for body weight or other anthropomorphic measures.) To maximize compliance, once-a-day dosing responses are simulated in Figures 5A-C for $<10 \%$ RTF, $10-20 \%$ RTF, and 
TABLE 3 | Thirteen trials of monotherapy vs. combination therapy, categorized according to whether patients experienced benefits or not during combination therapy

Benefit as assessed by improved outcomes

a) Substantial quality of life or mood or neurocognitive benefit

Bunevicius, 1999

Nygaard

b) Some quality of life or mood or neurocognitive benefit

Escobar-Morreale (5 $\mu \mathrm{g}$ T3)

Escobar-Morreale (7.5 $\mu \mathrm{g}$ T3)

Saravanan*

c) No quality of life or mood or neurocognitive benefit

Appelhof (T4 + T3, 10:1 ratio)

Appelhof (T4 + T3, 5:1 ratio)

Bunevicius, 2002

Clyde

Fadeyev

Kaminski

Rodriguez

Sawka

Siegmund

Walsh

*Showed benefit at 6 months but not at 12 months.

$>20 \%$ RTF. This should keep T3 excursions within the normal range, as shown in the figures. If individual patient clinical requirements warrant, the LT4 + LT3 dosages can be split in half and prescribed $2 \mathrm{x}$ a day, with smaller excursions in serum $\mathrm{T} 3$, as shown in Figure 5D.

$$
\begin{aligned}
\text { For RTFs }<10 \% \rightarrow & \rightarrow 100 \mu \mathrm{g} \mathrm{LT} 4+10-12.5 \mu \mathrm{g} \mathrm{LT} 3, \text { once daily } \\
\text { For RTFs } 10-20 \% \rightarrow & \rightarrow 100 \mu \mathrm{g} \mathrm{LT} 4+7.5-10 \mu \mathrm{g} \mathrm{LT} 3, \text { once daily } \\
\text { For RTFs }>20 \% \rightarrow & \text { LT4 }=87.5 \mathrm{ug}+7.5 \mathrm{ug} \text { LT3, once daily } \\
\text { For RTFs }>20 \% \rightarrow & \text { LT4 }=50 \% \text { of } 87.5 \mathrm{ug} \\
& +50 \% \text { of } 7.5 \mathrm{ug} \text { LT3, twice daily. }
\end{aligned}
$$

\section{DISCUSSION}

Our two working hypotheses are reasonably well-supported by our simulation data and comparative analysis with the data from the 13 combination trials. Evidently, these hypotheses are strongly intertwined, in a complex way, probably as a consequence of the tight, nonlinear couplings and homeostatic feedback effects among these well-regulated hormones. Importantly, the T3 (and T4) levels that can be achieved during combination therapy, and whether they are low, medium or high, appear to be affected endogenously by RTF-in a nonlinear way-as well as by the exogenous LT4 and LT3 dosages given. The latter are immediately under the influence of the same endogenous regulatory system components following absorption of the dosages. Overall, if the RTF is low, the added T3 seems to provide more impact in terms of either improved outcomes or patient preference. If the RTF is high,
TABLE 4 | Seven trials of monotherapy vs. combination therapy, categorized according to whether patients preferred combination therapy or not.

\section{Therapeutic preference}

a) Preference for combination therapy

Appelhof (T4 + T3, 10:1 ratio)

Appelhof (T4 + T3, 5:1 ratio)

Bunevicius, 1999

Bunevicius, 2002

Escobar-Morreale (5 $\mu \mathrm{g}$ T3)

Escobar-Morreale (7.5 $\mu \mathrm{g}$ T3)

Nygaard

b) No preference for combination therapy

Saravanan

Walsh

the same dose of T3 appears to have less impact However, if the amount of T3 added is relatively high, thus achieving a high or supraphysiologic T3 level, then there also is a positive impact in terms of either improved outcomes or patient preference-with due consideration to the clinical effects of T3 toxicity.

We recognized in existing trial data that, in the presence of sufficient T4, the T3 levels needed to ensure patient preference were higher than those needed to provide improved outcome measures; and this was borne out by our analyses. This motivated our coupled hypotheses and their analysis by "whatif" simulations of the trial data. We found a similar number of studies (five studies) associated with patient preference for combination therapy $(9-11,13,16)$ as those demonstrating improved outcomes (four studies) $(11,13,16,18)$. Four of the five studies that showed patient preference had high simulated (and measured) T3 levels $(9-11,16)$, the exception being the Escobar-Morreale study (13) in which the simulated T3 levels were mid-range.

Our analysis was limited by several complicating factors present or absent in the trial data. T4/T3 ratios reported in the various studies were very different, some with initially higher $\mathrm{T} 4 / \mathrm{T} 3$ ratios at baseline and the T4/T3 ratios substantially lower in the combination therapy arm $(15,17)$. In addition, not all studies provided full laboratory values at baseline, during the study, or at the end of the study [e.g., Nygaard (16)]. In a few studies dosing regimens were not clear. One study (18) showed improved outcomes at 6 months, but not at 12 months. We classified this study as having a positive outcome, in part because all other studies were 6 months or less in duration. However, this may not be the best way to categorize this trial, which may have demonstrated a placebo effect at 6 months. The 2002 Bunevicius study (6) was not amenable to comparative analysis as the RTF appeared to be $>100 \%$; this might be because this hypothyroid trial population consisted of Graves' disease patients who had undergone surgery for their disease, which may have been incomplete, with enough residual thyroid tissue to make dosing formulation more difficult. The Valizadeh study (21) could not be simulated for unclear reasons. 

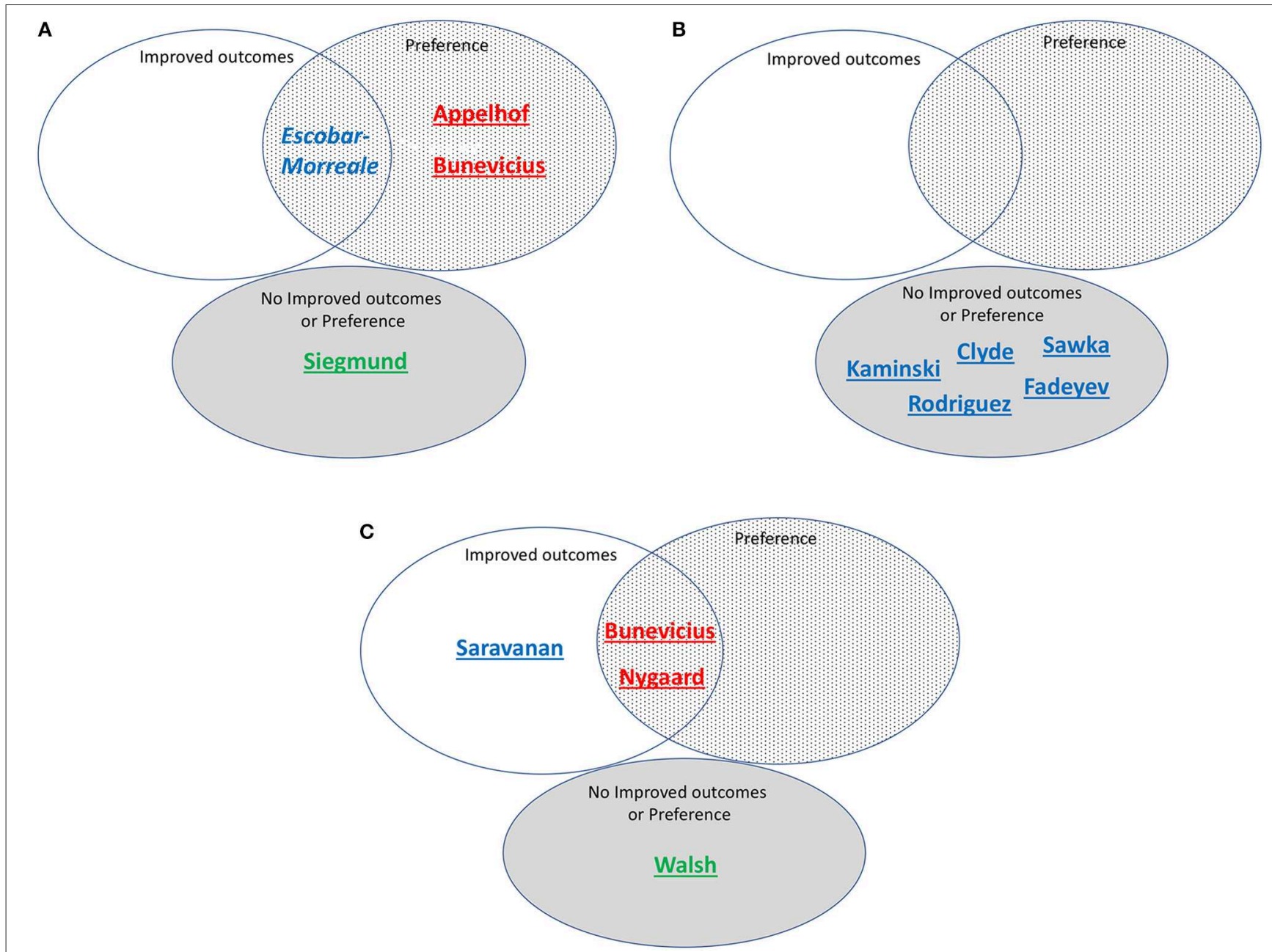

FIGURE 4 | (A) Studies assessed as including patients with high residual thyroid function (>20\%) and/or T3 levels predicted by simulation, grouped according to whether improved outcomes, preference, both improved outcomes and preference, or neither improved outcomes nor preference was reported. Red font indicates high simulated T3 levels. Blue font indicates medium simulated T3 levels. Green font indicates low simulated T3 levels. Underlined indicates correctly predicted. Italics indicate incorrect prediction. Bunevicius is 2002 study. (B) Studies assessed as including patients with medium residual thyroid function (10-20\%) and/or T3 levels predicted by simulation, grouped according to whether improved outcomes, preference, both improved outcomes and preference, or neither improved outcomes nor preference was reported. Red font indicates high simulated T3 levels. Blue font indicates medium simulated T3 levels. Underlined indicates correctly predicted. (C) Studies assessed as including patients with low residual thyroid function (<10\%) and/or T3 levels predicted by simulation, grouped according to whether improved outcomes, preference, both improved outcomes and preference, or neither improved outcomes nor preference was reported. Red font indicates high simulated T3 levels. Blue font indicates medium simulated T3 levels. Green font indicates low simulated T3 levels. Underlined indicates correctly predicted. Bunevicius is 1999 study.

Additional limitations of prior studies that might have affected the rigor of our analyses include the following. The studies clearly included patients with different etiologies of their hypothyroidism and a wide spectrum of RTF values. There is inter-assay variability across the various studies conducted in various countries, especially for FT3 assays, making it difficult to obtain very close comparative results in all cases. Not all studies reported the timing of phlebotomy, and whether blood samples were drawn at random times of day or were trough levels, making it possible that thyroid hormone levels, particularly T3 or FT3 could vary by as much as $40 \%$ (34).
There are also limitations of the trials that, in turn, may have led to limitations in our analysis of them. With regard to patient satisfaction and patient preference issues assessed in the various trials, it must be acknowledged that many symptoms of hypothyroidism are non-specific and overlap with symptoms of other conditions (35). Therefore, it is possible that lack of improvement in quality of life, mood, or neurocognitive function noted could have occurred because reported deficits were not thyroid-related. It is also possible that improvements in mood or preference for combination therapy were reported because a different condition such as depression was in fact being treated $(36,37)$. In addition, some of these trials 

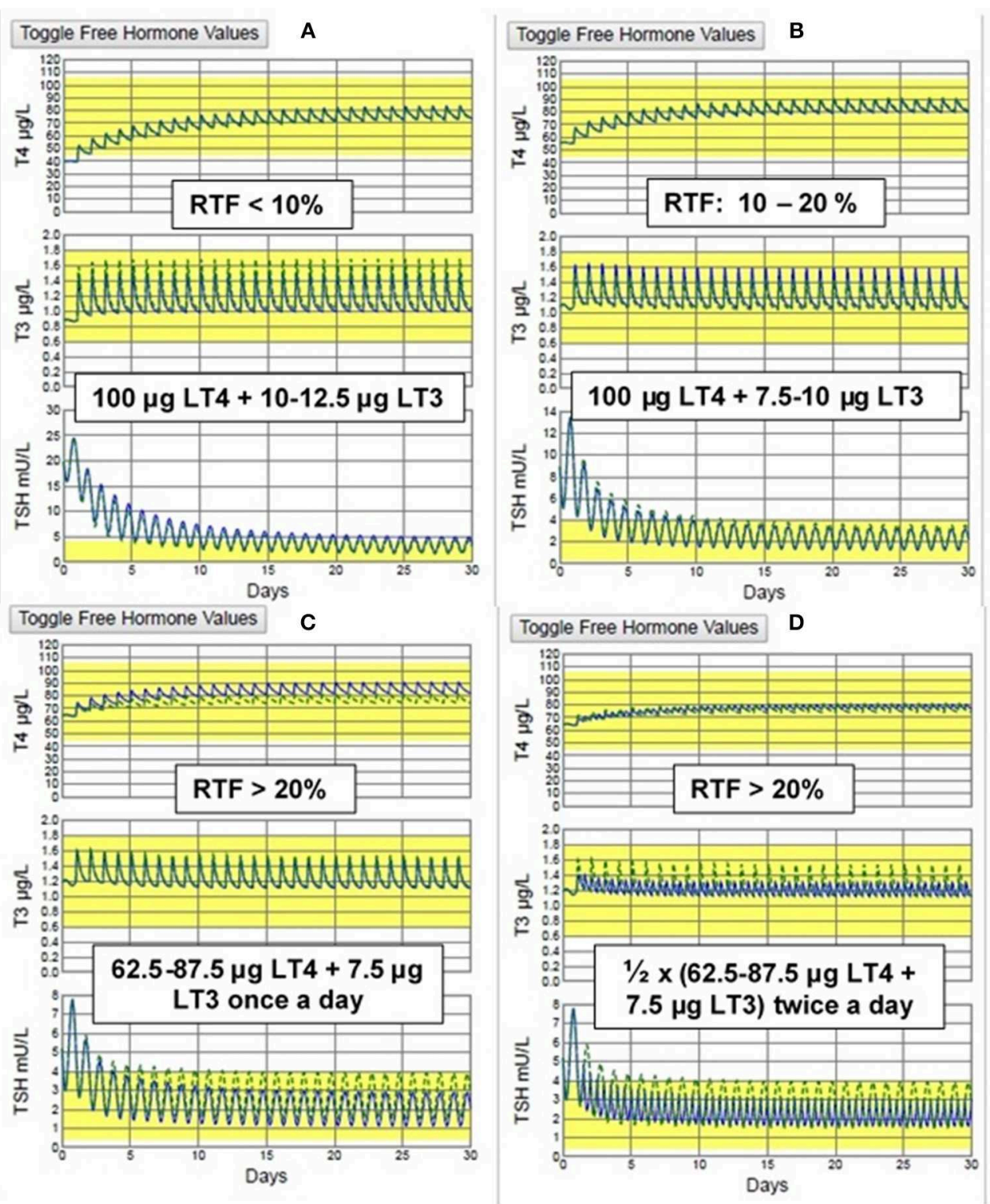

FIGURE 5 | THYROSIM simulated T4, T3, \& TSH responses to the recommended dosage combinations: for (A) low RTF (<10\%) \& (B) medium RTF (10-20\%) (TOP) \& (C) high (>20\%) RTF using once daily dosing and (D) high (>20\%) RTF using twice a day dosing. (BOTTOM). The smallest T3 (and TSH) excursions are seen with twice daily dosing, but no values are outside the normal ranges with once a day dosing.

may have been too short to allow sufficient adaptation for either benefits to be seen or adverse effects to occur. Despite these limitations, we believe we have achieved the goal of our studies.

In summary, our results reliably support the notion that RTF differences are a key factor in explaining the ambiguities in the spectrum of combination therapy study results reported between 1999 and 2016. As added value, we have adapted our RTF estimation methodology for combined LT4 + LT3 dosing that is practical and potentially optimal when designing a combination therapy trial. Serum TSH, T4 and/or T3 levels at the time of diagnosis should be obtained from patient history data or anew, and prior to initiating any therapy; and one or more of the three graphs in Figure 3 can then be used as nomograms to estimate RTF from individual patient data. Using this algorithm, we have provided combination dosing schemes that should serve best for starting dosing in $70 \mathrm{~kg}$ individuals with computed RTFs in the three given ranges. These are readily scaled by individual patient requirements, body weights or other anthropomorphic measurements.

\section{DATA AVAILABILITY STATEMENT}

The datasets generated for this study are available on request to the corresponding author. 


\section{AUTHOR CONTRIBUTIONS}

All authors listed have made a substantial, direct and intellectual contribution to the work, and approved it for publication.

\section{REFERENCES}

1. Peterson SJ, Cappola AR, Castro MR, Dayan CM, Farwell AP, Hennessey $\mathrm{JV}$, et al. An online survey of hypothyroid patients demonstrates prominent dissatisfaction. Thyroid. (2018) 28:707-21. doi: 10.1089/thy.2017.0681

2. Jonklaas J, Tefera E, Shara N. Prescribing therapy for hypothyroidism: influence of physician characteristics. Thyroid. (2019) 29:44-52. doi: 10.1089/thy.2018.0369

3. Jonklaas J, Tefera E, Shara N. Physician choice of hypothyroidism therapy: influence of patient characteristics. Thyroid. (2018) 28:1416-24. doi: $10.1089 /$ thy. 2018.0325

4. Jonklaas J, Tefera E, Shara N. Short-term time trends in prescribing therapy for hypothyroidism: results of a Survey of American Thyroid Association Members. Front Endocrinol. (2019) 10:31. doi: 10.3389/fendo.2019.00031

5. Jonklaas J, Davidson B, Bhagat S, Soldin SJ. Triiodothyronine levels in athyreotic individuals during levothyroxine therapy. JAMA. (2008) 299:76977. doi: 10.1001/jama.299.7.769

6. Gullo D, Latina A, Frasca F, Le Moli R, Pellegriti G, Vigneri R. Levothyroxine monotherapy cannot guarantee euthyroidism in all athyreotic patients. PLoS ONE. (2011) 6:e22552. doi: 10.1371/journal.pone.0022552

7. Escobar-Morreale HF, del Rey FE, Obregon MJ, de Escobar GM. Only the combined treatment with thyroxine and triiodothyronine ensures euthyroidism in all tissues of the thyroidectomized rat. Endocrinology. (1996) 137:2490-502. doi: 10.1210/endo.137.6.8641203

8. Werneck de Castro JP, Fonseca TL, Ueta CB, McAninch EA, Abdalla S, Wittmann G, et al. Differences in hypothalamic type 2 deiodinase ubiquitination explain localized sensitivity to thyroxine. J Clin Invest. (2015) 125:769-81. doi: 10.1172/JCI77588

9. Appelhof BC, Fliers E, Wekking EM, Schene AH, Huyser J, Tijssen JG, et al. Combined therapy with levothyroxine and liothyronine in two ratios, compared with levothyroxine monotherapy in primary hypothyroidism: a double-blind, randomized, controlled clinical trial. J Clin Endocrinol Metab. (2005) 90:2666-74. doi: 10.1210/jc.2004-2111

10. Bunevicius R, Jakuboniene N, Jurkevicius R, Cernicat J, Lasas L, Prange AJ Jr. Thyroxine vs thyroxine plus triiodothyronine in treatment of hypothyroidism after thyroidectomy for Graves' disease. Endocrine. (2002) 18:129-33. doi: 10.1385/ENDO:18:2:129

11. Bunevicius R, Kazanavicius G, Zalinkevicius R, Prange AJ Jr. Effects of thyroxine as compared with thyroxine plus triiodothyronine in patients with hypothyroidism. N Engl J Med. (1999) 340:424-9. doi: 10.1056/NEJM199902113400603

12. Clyde PW, Harari AE, Getka EJ, Shakir KM. Combined levothyroxine plus liothyronine compared with levothyroxine alone in primary hypothyroidism: a randomized controlled trial. JAMA. (2003) 290:2952-8. doi: 10.1001/jama.290.22.2952

13. Escobar-Morreale HF, Botella-Carretero JI, Gomez-Bueno M, Galan JM, Barrios V, Sancho J. Thyroid hormone replacement therapy in primary hypothyroidism: a randomized trial comparing L-thyroxine plus liothyronine with L-thyroxine alone. Ann Intern Med. (2005) 142:412-24. doi: 10.7326/0003-4819-142-6-200503150-00007

14. Fadeyev VV, Morgunova TB, Melnichenko GA, Dedov, II. Combined therapy with L-thyroxine and L-triiodothyronine compared to L-thyroxine alone in the treatment of primary hypothyroidism. Hormones. (2010) 9:245-52. doi: 10.14310/horm.2002.1274

15. Kaminski J, Miasaki FY, Paz-Filho G, Graf H, Carvalho GA. Treatment of hypothyroidism with levothyroxine plus liothyronine: a randomized, double-blind, crossover study. Arch Endocrinol Metab. (2016) 60:562-72. doi: 10.1590/2359-3997000000192

16. Nygaard B, Jensen EW, Kvetny J, Jarlov A, Faber J. Effect of combination therapy with thyroxine (T4) and 3,5,3'-triiodothyronine versus T4

\section{FUNDING}

$\mathrm{JJ}$ is supported by grants 1R01DE025822 from NIDCR and 5UL1TR001409 from NCATS. monotherapy in patients with hypothyroidism, a double-blind, randomised cross-over study. Eur J Endocrinol. (2009) 161:895-902. doi: 10.1530/EJE-09-0542

17. Rodriguez T, Lavis VR, Meininger JC, Kapadia AS, Stafford LF. Substitution of liothyronine at a 1:5 ratio for a portion of levothyroxine: effect on fatigue, symptoms of depression, and working memory versus treatment with levothyroxine alone. Endocr Pract. (2005) 11:223-33. doi: 10.4158/EP.11.4.223

18. Saravanan P, Simmons DJ, Greenwood R, Peters TJ, Dayan CM. Partial substitution of thyroxine (T4) with tri-iodothyronine in patients on T4 replacement therapy: results of a large community-based randomized controlled trial. J Clin Endocrinol Metab. (2005) 90:805-12. doi: $10.1210 /$ jc.2004-1672

19. Sawka AM, Gerstein HC, Marriott MJ, MacQueen GM, Joffe RT. Does a combination regimen of thyroxine (T4) and 3,5,3'-triiodothyronine improve depressive symptoms better than T4 alone in patients with hypothyroidism? Results of a double-blind, randomized, controlled trial. J Clin Endocrinol Metab. (2003) 88:4551-5. doi: 10.1210/jc.2003-030139

20. Siegmund W, Spieker K, Weike AI, Giessmann T, Modess C, Dabers T, et al. Replacement therapy with levothyroxine plus triiodothyronine (bioavailable molar ratio $14: 1)$ is not superior to thyroxine alone to improve well-being and cognitive performance in hypothyroidism. Clin Endocrinol. (2004) 60:750-7. doi: $10.1111 / \mathrm{j} .1365-2265.2004 .02050 . \mathrm{x}$

21. Valizadeh M, Seyyed-Majidi MR, Hajibeigloo H, Momtazi S, Musavinasab N, Hayatbakhsh MR. Efficacy of combined levothyroxine and liothyronine as compared with levothyroxine monotherapy in primary hypothyroidism: a randomized controlled trial. Endocr Res. (2009) 34:80-9. doi: 10.1080/07435800903156340

22. Walsh JP, Shiels L, Lim EM, Bhagat CI, Ward LC, Stuckey BG, et al. Combined thyroxine/liothyronine treatment does not improve well-being, quality of life, or cognitive function compared to thyroxine alone: a randomized controlled trial in patients with primary hypothyroidism. J Clin Endocrinol Metab. (2003) 88:4543-50. doi: 10.1210/jc.2003-030249

23. Jonklaas J, Bianco AC, Bauer AJ, Burman KD, Cappola AR, Celi FS, et al. Guidelines for the treatment of hypothyroidism: prepared by the american thyroid association task force on thyroid hormone replacement. Thyroid. (2014) 24:1670-751. doi: 10.1089/thy.2014.0028

24. Joffe RT, Brimacombe M, Levitt AJ, Stagnaro-Green A. Treatment of clinical hypothyroidism with thyroxine and triiodothyronine: a literature review and metaanalysis. Psychosomatics. (2007) 48:379-84. doi: 10.1176/appi.psy.48.5.379

25. Grozinsky-Glasberg S, Fraser A, Nahshoni E, Weizman A, Leibovici L. Thyroxine-triiodothyronine combination therapy versus thyroxine monotherapy for clinical hypothyroidism: meta-analysis of randomized controlled trials. J Clin Endocrinol Metab. (2006) 91:2592-9. doi: 10.1210/jc.2006-0448

26. Ma C, Xie J, Huang X, Wang G, Wang Y, Wang X, et al. Thyroxine alone or thyroxine plus triiodothyronine replacement therapy for hypothyroidism. Nucl Med Commun. (2009) 30:586-93. doi: 10.1097/MNM.0b013e32832c79e0

27. http://biocyb1.cs.ucla.edu/thyrosim/

28. Eisenberg M, Samuels M, DiStefano JJ III. Extensions, validation, and clinical applications of a feedback control system simulator of the hypothalamo-pituitary-thyroid axis. Thyroid. (2008) 18:1071-85. doi: $10.1089 /$ thy.2007.0388

29. Eisenberg M, Samuels M, DiStefano JJ III. L-T4 bioequivalence and hormone replacement studies via feedback control simulations. Thyroid. (2006) 16:1279-92. doi: 10.1089/thy.2006.16.1279

30. Ben-Shachar R, Eisenberg M, Huang SA, DiStefano JJ III. Simulation of post-thyroidectomy treatment alternatives for triiodothyronine or thyroxine replacement in pediatric thyroid cancer patients. Thyroid. (2012) 22:595-603. doi: $10.1089 /$ thy.2011.0355 
31. Eisenberg MC, Santini F, Marsili A, Pinchera A, DiStefano JJ III. TSH regulation dynamics in central and extreme primary hypothyroidism. Thyroid. (2010) 20:1215-28. doi: 10.1089/thy.2009.0349

32. Eisenberg M, Distefano JJ. TSH-based protocol, tablet instability, and absorption effects on L-T4 bioequivalence. Thyroid. (2009) 19:103-10. doi: 10.1089/thy.2008.0148

33. Han SX, Eisenberg M, Larsen PR, DiStefano J III. THYROSIM App for education and research predicts potential health risks of over-thecounter thyroid supplements. Thyroid. (2016) 26:489-98. doi: 10.1089/thy.201 5.0373

34. Saravanan P, Siddique H, Simmons DJ, Greenwood R, Dayan CM. Twenty-four hour hormone profiles of TSH, Free T3 and free T4 in hypothyroid patients on combined T3/T4 therapy. Exp Clin Endocrinol Diabetes. (2007) 115:261-7. doi: 10.1055/s-2007973071

35. Jonklaas J. Persistent hypothyroid symptoms in a patient with a normal thyroid stimulating hormone level. Curr Opin Endocrinol Diabetes Obes. (2017) 24:356-63. doi: 10.1097/MED.00000000000 00355
36. Duntas LH, Maillis A. Hypothyroidism and depression: salient aspects of pathogenesis and management. Minerva Endocrinol. (2013) 38:365-77.

37. Parmentier T, Sienaert P. The use of triiodothyronine (T3) in the treatment of bipolar depression: A review of the literature. J Affect Disord. (2018) 229:410-4. doi: 10.1016/j.jad.2017.12.071

Conflict of Interest: JD is the creator and developer of the THYROSIM app.

The remaining author declares that the research was conducted in the absence of any commercial or financial relationships that could be construed as a potential conflict of interest.

Copyright (C) 2019 DiStefano and Jonklaas. This is an open-access article distributed under the terms of the Creative Commons Attribution License (CC BY). The use, distribution or reproduction in other forums is permitted, provided the original author(s) and the copyright owner(s) are credited and that the original publication in this journal is cited, in accordance with accepted academic practice. No use, distribution or reproduction is permitted which does not comply with these terms. 